\title{
Pharmacological manipulation of serotonin receptors during brain embryogenesis favours stress resiliency in female rats
}

\author{
Gianluca Lavanco, ${ }^{1}$ Angela Cavallaro, ${ }^{1}$ Emanuele Cannizzaro, ${ }^{1}$ Marco Giammanco, ${ }^{2}$ Danila Di Majo, ${ }^{2}$ \\ Anna Brancato ${ }^{1}$ \\ ${ }^{1}$ Department of Sciences for Health Promotion and Mother and Child Care Giuseppe D'Alessandro, University of \\ Palermo; ${ }^{2}$ Department of Experimental Biomedicine and Clinical Neurosciences, University of Palermo, Palermo, Italy
}

\begin{abstract}
Manipulations of the serotonin transmission during early development induce long-lasting changes in the serotonergic circuitry throughout the brain. However, little is known on the developmental consequences in the female progeny. Therefore, this study aimed at exploring the behavioural effects of pre- and postnatal stimulation of the serotonergic system by 5-methoxytryptamine in adolescent female rats on behavioural reactivity and anxiety-like phenotype. Our results show that perinatal 5methoxythyptamine decreased total distance travelled and rearing frequency in the novel enviroment, and increased the preference for the centre of the arena in the open field test. Moreover, perinatal 5-methoxytryptamine increased the percentages of entries and time spent on the open arms of the elevated plus maze, with respect to perinatally vehicle-exposed rats. Thus, perinatal stimulation of serotonin receptors does not impair the functional response to the emotional challenges in female rats, favouring the occurrence of a stress-resilient phenotype.
\end{abstract}

\footnotetext{
Correspondence: Anna Brancato, Department of Sciences for Health Promotion and Mother and Child Care Giuseppe D'Alessandro, University of Palermo, via del Vespro 129, 90127 Palermo, Italy Tel.: +39.0916555855.

E-mail: anna.brancato@unipa.it
}

Key words: serotonin; 5-methoxytryptamine; development; stress reactivity; female rats.

Conflict of interest: the authors declare no potential conflict of interest.

Contributions: the authors contributed equally.

Received for publication: 8 May 2017.

Revision received: 5 June 2017.

Accepted for publication: 15 June 2017.

(C) Copyright G. Lavanco et al., 2017

Licensee PAGEPress, Italy

Journal of Biological Research 2017; 90:6782

doi:10.4081/jbr.2017.6782

This article is distributed under the terms of the Creative Commons Attribution Noncommercial License (by-nc 4.0) which permits any noncommercial use, distribution, and reproduction in any medium, provided the original author(s) and source are credited.

\section{Introduction}

It is well known that the exposure to traumatic events, or pharmacological treatments, at different life stages, and especially during the early gestational period, induces dysfunction in several areas of the central nervous system. ${ }^{1-8}$ In particular, disturbances in the serotonergic transmission seem to play a crucial role in the onset of the detrimental consequences of psychopathological traits that appear often in adolescence. ${ }^{9}$ Recent investigations show that manipulation of serotonin (5-hydroxytriptamine or 5-HT) during early development induce long-lasting changes in the serotonergic circuitry throughout the brain. In this regard, maternal buspirone, a 5-HT1A-receptor agonist, has been shown to protect against the adverse effects of in utero stress on pain sensitivity in adult rat offspring, likely due to the interplay between 5-HT and neuropeptidergic systems involved in nociception. ${ }^{10-15}$ A number of studies on the effects of prenatal serotonin agonists on receptor development employed the 5-HT1/5-HT2 agonist 5-methoxytryptamine (5-MT) and produced considerable insight into the ability of preand post-natal pharmacological manipulation of 5-HT receptors to decrease behavioural reactivity to stress in pups and facilitate learning performance in adolescent male rats. ${ }^{16-19}$ Notably, adolescence represents a vulnerable phase of development when the serotonergic system is still maturing and the influence of the sexual hormones plays a role in determining attitudes and behaviour.

Notwithstanding, little preclinical evidence regards the consequences of the perinatal manipulation of the serotonergic system in the female progeny. Therefore the present study aimed at investigating the behavioural consequences of pre- and post-natal stimulation of the serotonergic system by 5-MT. In particular, behavioural reactivity to novelty, locomotor activity and anxiety-like phenotype were assessed, in order to shed light on putative gender-related peculiarities in the occurrence of psychopathological traits associated to manipulations of the serotonergic transmission in critical period of ontogenesis.

\section{Materials and Methods}

\section{Experimental procedures}

Wistar rats of both sexes (Harlan, Udine, Italy) housed with free access to food and water were maintained on a $12 \mathrm{~h}$ on/off cycle (8:00-20:00) at a constant temperature $\left(22 \pm 2^{\circ} \mathrm{C}\right)$ and humidity $(55 \pm 10 \%)$. Pairs of primiparous females of 120 days of age were mated with one male of 150 days of age. The day on which sperm was detected in the vaginal smear was designed as gesta- 
tional day (GD) 1 . Ten days before delivery 10 pregnant dam was individually housed in sawdust-containing plastic cages (40 $\mathrm{cm} \times 60 \mathrm{~cm}, 20 \mathrm{~cm}$ in height) and randomly assigned to the gestational pharmacological exposure ( $\mathrm{n}=5$ per group).

Perinatal exposure to vehicle or 5-MT was designed as follows: pregnant dams received a single daily injection of vehicle or 5-MT (1mg/kg s.c.) over GD 12-21, time in which 5-HT1A receptors appear in the foetal brain. All litters born within a 2-day period were reduced to eight pups (four males and four females), and maintained under standard laboratory conditions. Two days after birth, female offspring received a daily injection of vehicle or 5MT $(0.5 \mathrm{mg} / \mathrm{kg}$ s.c.) from postnatal day (PND) 2 until PND 18, when 5-HT1A receptors reach their maturation. ${ }^{19}$ The dosage was reduced in order to minimise possible adverse neurotoxic effects due to the immature metabolic system of pups. Immediately after birth and at weaning time, no significant differences in number, morbidity, mortality or weight were observed between the perinatally vehicle- and the perinatally 5-MT-exposed offspring. On weaning time (PND 24), according to the perinatal exposure and the postnatal acute treatments, female pups were randomly assigned to the following two experimental groups $(n=18)$; perinatal vehicle (VH) and perinatal 5-MT (5-MT). On the test day (PND 42), adolescent rats were brought into the laboratory $60 \mathrm{~min}$ before the experimental sessions to acclimatize (Figure 1). The experiments were carried out in a sound isolated room between 9:00 and 17:00. Animal performance was recorded on videotape and monitored in an adjacent room. To ensure that rat's behaviour was not affected by the detection of another rats' scent, all the devices were thoroughly cleaned $10 \mathrm{~min}$ before the animal's entry into the cages. All the experiments were conducted in accordance with the regulations of the Committee for the Protection and Use of Animals of the University of Palermo, in accordance with current Italian legislation on animal experimentation (D.L. 116/92) and the European directives $(2010 / 63 / \mathrm{EU})$ on care and use of laboratory animals. Every effort was made to minimize the number of animals used and their sorrow.

\section{Open field test}

Rats were first tested in an open field arena. Locomotor activity and behavioural reactivity in a novel environment were measured into a dimly illuminated (100 lx) chamber with an automatic video-tracking system, Any Maze (Ugo Basile, Italy). The apparatus consisted in a Plexiglas square box $(44 \times 44 \times 20 \mathrm{~cm})$. The system produces a quali-quantitative mapping of the motor patterns. Different parameters were measured simultaneously: total distance travelled, rearing frequency and central zone Preference. Each experimental session lasted $5 \mathrm{~min}$.

\section{Elevated plus-maze test}

Rats were tested into a mean light intensity (100 lx) illuminated chamber. The elevated plus maze was made from dark grey PVC and consisted of a plus-shaped platform elevated to a height of $70 \mathrm{~cm}$ above the floor. Two of the opposing arms $(55 \times 10 \mathrm{~cm})$ were closed by $40 \mathrm{~cm}$-high walls (closed arms) whereas the other two arms had no walls (open arms). All four arms were connected at the centre by $10 \times 10$ platform. At the beginning of each session, rats were placed in the centre of the maze facing one of the open arms. The number of entries and the time spent on the open and closed arms were recorded by using the ANY MAZE Video Tracking System, for 5 min. Entries were scored when all the four paws entered into each single arm. Results were expressed as percentage of entries and time spent on the open arms/open+closed arms.

\section{Statistical analysis}

Open field test: locomotor activity evaluation was conducted performing a two-tailed Student's t-test for unpaired measures on total distance travelled. Behavioural reactivity to novelty was analysed by performing a two-tailed Student's t-test for unpaired measures on rearing frequency and central zone Preference.

Elevated plus maze: the analysis of the anxiety-like behaviour was carried out using a two-tailed Student's t-test for unpaired measure on the percentage of the entries in the open arms/the entries in the open and closed arms, and as the percentage of the time spent on the open arms/the time spent on the open and closed arms.

\section{Results}

\section{Open field test}

Female rats were first tested in an open field arena at PND 42 in order to assess whether perinatal exposure to 5-MT-induced differences in the measured parameters: total distance travelled, rearing frequency and central zone Preference compared to perinatally vehicle-exposed rats. Statistical analysis by a two-tailed Student's t-test for unpaired measure showed that perinatal exposure to 5MT reduced total distance travelled $(\mathrm{t}=3.830, \mathrm{df}=34 ; \mathrm{P}=0.0005)$ and rearing frequency $(\mathrm{t}=2.999, \mathrm{df}=34 ; \mathrm{P}=0.0050)$ and increased central zone Preference $(\mathrm{t}=2.276, \mathrm{df}=34 ; \mathrm{P}=0.0293)$ compared to perinatally vehicle-exposed offspring (Figure 2).

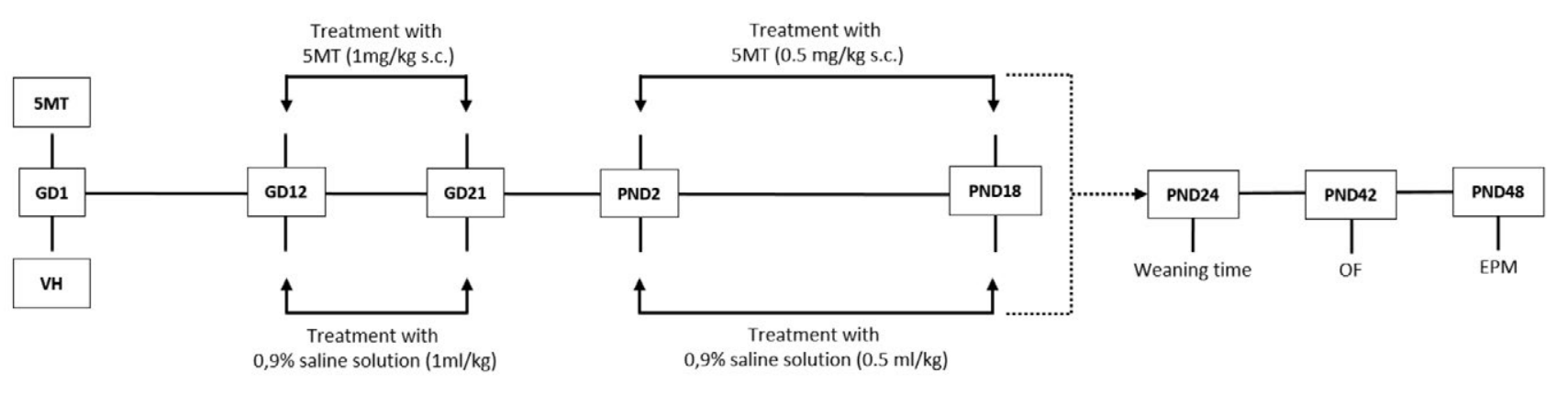

Figure 1. Timeline showing days and time on which treatment and behavioural testing were conducted. 5-MT: 5-methoxytryptamine, EPM: elevated plus maze, GD: gestational day, OF: open field, PND: postnatal day, VH: vehicle. 


\section{Elevated plus-maze test}

Female rats were tested in elevated plus maze at PND 48 in order to evaluate the effects of perinatal exposure to 5-MT on anxiety-like behaviour, expressed as the preference for the open arms. Statistical analysis by a two-tailed Student's t-test for unpaired measure showed that perinatal exposure to 5-MT increased the percentage of the time spent on the open arms/the time spent on the open and closed arms $(t=2.999, \mathrm{df}=34 ; \mathrm{P}=0.0050)$ and the percentage of the entries in the open arms/the entries in the open and closed arms $(\mathrm{t}=3.830, \mathrm{df}=34 ; \mathrm{P}=0.0005)$ compared to perinatally vehicle-exposed offspring (Figure 3).

\section{Discussion and Conclusions}

The present study investigated in adolescent female rats the consequences of the perinatal pharmacological manipulation of the serotonergic system by a non-selective 5-HT receptor agonist, 5-MT, on behavioural reactivity and anxiety-like behaviour measured by the open field test and the elevated plus-maze, two well-validated unconditioned state anxiety models in rats. ${ }^{20-23}$ Our results show that, when exposed for the first time to the novel environment, perinatally 5-MT-treated female rats displayed lower exploratory activity in the open field than vehicle-treated counterparts: this was shown by reduced total distance travelled and rearing frequency. However, rats did not avoid the central zone of the open field, which is usually perceived as anxiogenic. Indeed, perinatally 5-MT-exposed female rats displayed an increased preference for the centre of the arena, where they spent longer time than perinatally vehicle-treated controls. Notably, when rats are tested for the first time in the open field, they commonly show high behavioural reactivity towards the new environment. Both total distance travelled and rearing frequency are generally increased, indicating an initial escape behaviour. ${ }^{24}$ At the same time, rats exhibit low exploratory activity in the centre of the arena, reflecting avoidance of an unprotected anxiogenic environment. This behaviour is reversed when rats show a low emotional response to stressful conditions or they are treated with anxiolytic drugs: this results in decreased total distance travelled and rearing frequency, together with a preference for the centre of the arena. ${ }^{24,25}$ When tested in the elevated plus maze, perinatally 5-MT-treated females displayed an increase in the percentage of entries and time spent on the open arms, compared to perinatally vehicle-exposed rats. The elevated plus maze represents a valid behavioural model to study the emotional response of animals. An avoidance or a preference for the unprotected stressful open arms are respectively ascribed to an increase or a reduction in state anxiety. Indeed, anxiogenic drugs increase the avoidance of the open arms, while anxiolytic drugs reduce it. ${ }^{26}$ Therefore, the high values in the percentage of entries and time spent on the open arms of the elevated plus maze, observed in perinatally 5-MT-exposed rats, suggest a lower emotional response to the stressful environment than perinatally vehicleexposed rats. This evidence appears to be consistent with what observed in the open field test confirming a reduced emotional response during non-aversive stress-related behavioural tasks. ${ }^{17,18,27,28}$ Moreover our data are in agreement with previous experiments showing that prenatally 5-MT-treated male rats displayed reduced stress reactivity, anxiety- and depression-like behaviour. Altogether, our evidence indicates that perinatally 5-MT exposure does not exert gender-related phenotypes in adolescence.

Notably, central serotonergic system is involved in the pathophysiology of anxiety and in the pharmacological response to anxiolytic drugs. Preclinical models of anxiety show that drugs able to reduce serotonergic function decrease fearful behaviour and display anxiolytic-like effects in rats. ${ }^{29}$ The effects of serotonin on the physiological and behavioural responses to stressful stimuli seem to be dependent on the receptor subtype where it acts upon. Among the multiple subtypes of receptors for serotonin in the brain, 5HT1A receptor plays a major role in the regulation of serotonin neuronal activity and it is a critical mediator of the neuroendocrine responses to stress. 5-HT1A receptors are located both postsynaptically and presinaptically, the latter mediating the anxiolytic effects. ${ }^{29}$ Moreover, 5-HT1A receptor is the earliest serotonin receptor subtype which appears during the brain development, both in humans and in rodents. ${ }^{30}$

Although the mechanism underlying 5-MT effects that we report in this study remains to be further elucidated, we currently favour the hypothesis that perinatal exposure to 5-MT does not deteriorate the functional response of 5-HT1A receptors. An imbalance between pre- and postsynaptic 5-HT1A signalling, in favour of the anxiolytic-like signal transduction, might be responsible for the stress-resilient phenotype observed as a result of the perinatal 5-MT exposure. ${ }^{17}$

Further studies will explore the molecular mechanisms by which serotonin elicits its effects on brain development in both genders.
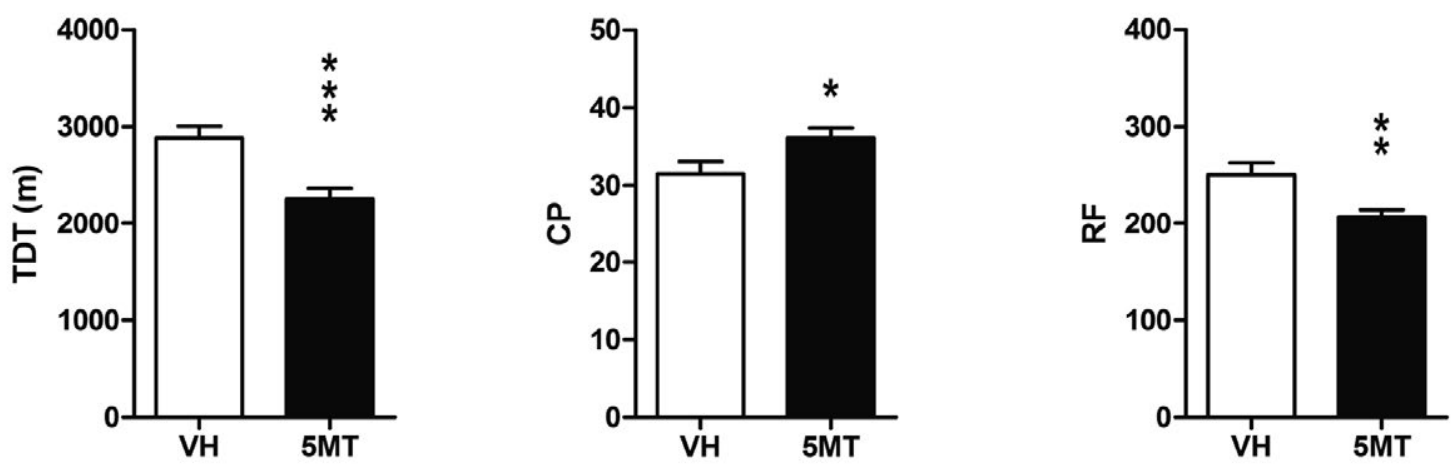

Figure 2. Open field test. Effects of perinatal 5-MT exposure on total distance travelled (TDT), rearing frequency (RF) and central preference $(\mathrm{CP})$ of the arena. Each value represents the mean \pm S.E.M. of eighteen rats. ${ }^{* * *} \mathrm{P}<0.001,{ }^{* *} \mathrm{P}<0.01,{ }^{*} \mathrm{P}<0.05$ vs perinatally vehicle-exposed rats. 

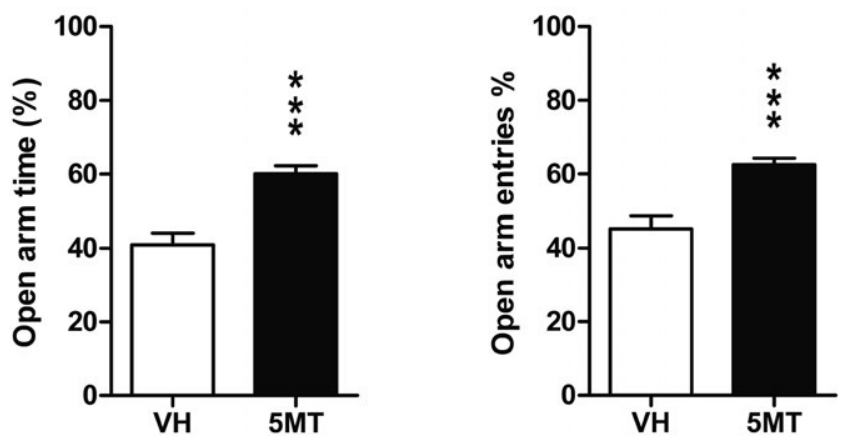

Figure 3. Elevated plus maze test. Effects of perinatal 5-MT exposure on percentage of entries and time spent on the open arms. Each value represents the mean \pm S.E.M. of eighteen rats. ${ }^{* * *} \mathrm{P}<0.001$ vs perinatally vehicle-exposed rats.

\section{References}

1. Brancato A, Plescia F, Lavanco G, et al. Continuous and intermittent alcohol free-choice from pre-gestational time to lactation: focus on drinking trajectories and maternal behavior. Front Behav Neurosci 2016;10:31.

2. Cacace S, Plescia F, Barberi I, Cannizzaro C. Acetaldehyde oral self-administration: evidence from the operant-conflict paradigm. Alcohol Clin Exp Res 2012;36:1278-87.

3. Rizzo V, Carletti F, Gambino G, et al. Role of CB2 receptors and cGMP pathway on the cannabinoid-dependent antiepileptic effects in an in vivo model of partial epilepsy. Epilepsy Res 2014; 108:1711-8.

4. Carletti F, Ferraro G, Rizzo V, et al. Antiepileptic effect of dimethyl sulfoxide in a rat model of temporal lobe epilepsy. Neurosci Lett 2013;546:31-5.

5. Cannizzaro E, Martire M, Gagliano M, et al. Reversal of prenatal diazepam-induced deficit in a spatial-object learning task by brief, periodic maternal separation in adult rats. Behav Brain Res 2005;161:320-30.

6. Cannizzaro C, Plescia F, Martire M, et al. Single intense prenatal stress decreases emotionality and enhances learning performance in the adolescent rat offspring: Interaction with a brief, daily maternal separation. Behav Brain Res 2006; 169:128-36.

7. Plescia F, Marino RA, Navarra M, et al. Early handling effect on female rat spatial and non-spatial learning and memory. Behav Processes 2014;103:9-16.

8. Cannizzaro E, Cannizzaro C, Plescia F, et al. Exposure to ototoxic agents and hearing loss: a review of current knowledge. Hearing Balance Commun 2014;12:166.

9. Van den Hove DL, Leibold NK, Strackx E, et al. Prenatal stress and subsequent exposure to chronic mild stress in rats; interdependent effects on emotional behavior and the serotonergic system. Eur Neuropsychopharmacol 2014;24:595-607.

10. Butkevich I, Mikhailenko V, Vershinina E, et al. Maternal buspirone protects against the adverse effects of in utero stress on emotional and pain-related behaviors in offspring. Physiol Behav 2011;102:137-42.

11. Xiao Y, Richter JA, Hurley JH. Release of glutamate and CGRP from trigeminal ganglion neurons: role of calcium channels and 5-HT1 receptor signaling. Mol Pain 2008;4:12.

12. Greco MC, Capuano A, Navarra P, Tringali G. Lacosamide inhibits calcitonin gene-related peptide production and release at trigeminal level in the rat. Eur J Pain 2016;20: 959-66.

13. Greco MC, Navarra P, Tringali G. The analgesic agent tapentadol inhibits calcitonin gene-related peptide release from isolated rat brainstem via a serotonergic mechanism. Life Sci 2016;145:161-5.

14. Greco MC, Lisi L, Currò D, et al. Tapentadol inhibits calcitonin gene-related peptide release from rat brainstem in vitro. Peptides. 2014;56:8-13.

15. Tringali G, Greco MC, Capuano A, et al. Flupirtine inhibits calcitonin-gene related peptide release from rat brainstem in vitro. Neurosci Lett 2012;506:332-5.

16. Kahne D, Tudorica A, Borella A, et al. Behavioral and magnetic resonance spectroscopic studies in the rat hyperserotonemic model of autism. Physiol Behav 2002;75:403-10.

17. Cannizzaro C, Plescia F, Gagliano M, et al. Effects of pre- and postnatal exposure to 5-methoxytryptamine and early handling on an object-place association learning task in adolescent rat offspring. Neurosci Res 2007;59:74-80.

18. Cannizzaro C, Plescia F, Gagliano M, et al. Perinatal exposure to 5-methoxytryptamine, behavioural-stress reactivity and functional response of 5-HT1A receptors in the adolescent rat. Behav Brain Res 2008;186:98-106.

19. Whitaker-Azmitia PM. Behavioural and cellular consequences of increasing serotonergic activity during brain development: a role in autism? Int J Dev Neurosci 2005;75-83.

20. Cannizzaro C, La Barbera M, Plescia F, et al. Ethanol modulates corticotropin releasing hormone release from the rat hypothalamus: does acetaldehyde play a role? Alcohol Clin Exp Res 2010;34:588-93.

21. Cannizzaro C, Plescia F, Cacace S. Role of acetaldehyde in alcohol addiction: current evidence and future perspectives. Malta Med J 2011;23.

22. Cacace S, Plescia F, La Barbera M, Cannizzaro C, Evaluation of chronic alcohol self-administration by a 3-bottle choice paradigm in adult male rats. Effects on behavioural reactivity, spatial learning and reference memory. Behavioural Brain Research 2011;219:213-20.

23. Plescia F, Brancato A, Venniro M, et al. Acetaldehyde selfadministration by a two-bottle choice paradigm: consequences on emotional reactivity, spatial learning, and memory. Alcohol 2015;49:139-48.

24. Aulich D. Escape versus exploratory activity: an interpretation of rats' behaviour in the open field and a light-dark preference test. Behav Proc 1976;1:153-64.

25. Brancato A, Plescia F, Marino RA, et al. Involvement of dopamine D2 receptors in addictive-like behaviour for acetaldehyde. PLoS One 2014;9:e99454.

26. Plescia F, Cannizzaro E, Brancato A, et al. Acetaldehyde effects in the brain. Acta Med Mediterr 2015;31:813-17.

27. Harris SS, Maciag D, Simpson KL, et al. Dose-dependent effects of neonatal SSRI exposure on adult behavior in the rat. Brain Res 2012;1429:52-60.

28. Lisboa SF, Oliveira PE, Costa LC, et al. Behavioral evaluation of male and female mice pups exposed to fluoxetine during pregnancy and lactation. Pharmacology 2007;80:49-56.

29. Maurel Remy S, Schreiber R, Dalmus M, De Vry J. Somatodendritic 5-HT1A receptors are critically involved in the anxiolytic effects of 8-OH-DPAT. Psychopharmacology 1996;125:89-91.

30. Patel TD, Zhou FC. Ontogeny of 5-HT1A receptor expression in the developing hippocampus. Brain Res Dev Brain Res 2005; 157:42-57. 